\section{(6) OPEN ACCESS}

\title{
Late mortality after acute hypoxic respiratory failure
}

\author{
Hallie C Prescott, ${ }^{1,2,3}$ Michael W Sjoding, ${ }^{1,2}$ Kenneth M Langa, 1,2,3,4 \\ Theodore J Iwashyna, 1,2,3,4 Daniel F McAuley ${ }^{5}$
}

- Additional material is published online only. To view please visit the journal online (http://dx.doi.org/10.1136/ thoraxjnl-2017-210109).

\section{${ }^{1}$ Department of Internal} Medicine, University of Michigan, Ann Arbor, Michigan, USA

${ }^{2}$ Institute for Healthcare Policy \& Innovation, University of Michigan, Ann Arbor, Michigan, USA

${ }^{3}$ VA Center for Clinical Management Research, HSR\&D Center of Innovation, Ann Arbor, Michigan, USA

${ }^{4}$ Institute for Social Research, Ann Arbor, Michigan, USA ${ }^{5}$ Department of Dentistry, and Biomedical Sciences, Queen's University of Belfast, Belfast, UK

\section{Correspondence to}

Dr Hallie C Prescott, Department of Internal Medicine, University of Michigan, 2800 Plymouth Road, North Campus Research Center, Building 16, 341E, Ann Arbor, MI 48109-2800, USA; hprescot@med.umich.edu

TJ and DFMA contributed equally.

Received 8 February 2017 Revised 9 June 2017 Accepted 3 July 2017 Published Online First 5 August 2017

\section{ABSTRACT}

Background Acute hypoxic respiratory failure (AHRF) is associated with significant acute mortality. It is unclear whether later mortality is predominantly driven by preexisting comorbid disease, the acute inciting event or is the result of AHRF itself.

Methods Observational cohort study of elderly US Health and Retirement Study (HRS) participants in fee-for-service Medicare (1998-2012). Patients hospitalised with AHRF were matched 1:1 to otherwise similar adults who were not currently hospitalised and separately to patients hospitalised with acute inciting events (pneumonia, non-pulmonary infection, aspiration, trauma, pancreatitis) that may result in AHRF, here termed at-risk hospitalisations. The primary outcome was late mortality-death in the 31 days to 2 years following hospital admission.

Results Among 15075 HRS participants, we identified 1268 AHRF and 13117 at-risk hospitalisations. AHRF hospitalisations were matched to 1157 non-hospitalised adults and 1017 at-risk hospitalisations. Among patients who survived at least 30 days, AHRF was associated with a $24.4 \%(95 \% \mathrm{Cl} 19.9 \%$ to $28.9 \%, \mathrm{p}<0.001)$ absolute increase in late mortality relative to adults not currently hospitalised and a $6.7 \%(95 \% \mathrm{Cl} 1.7 \%$ to $11.7 \%, p=0.01$ ) increase relative to adults hospitalised with acute inciting event(s) alone. At-risk hospitalisation explained $71.2 \%$ of the increased odds of late mortality, whereas the development of AHRF itself explained $28.8 \%$. Risk for death was equivalent to at-risk hospitalisation beyond 90 days, but remained elevated for more than 1 year compared with non-hospitalised controls.

Conclusions In this national sample of older Americans, approximately one in four survivors with AHRF had a late death not explained by pre-AHRF health status. More than $70 \%$ of this increased risk was associated with hospitalisation for acute inciting events, while $30 \%$ was associated with hypoxemic respiratory failure.

\section{INTRODUCTION}

Acute hypoxic respiratory failure (AHRF) is a leading cause of intensive care unit admission ${ }^{12}$ with high acute mortality. ${ }^{1}$ However, the impact of AHRF on late mortality (death in the 31 days to 2 years after hospital admission) is unclear. Studies with longer-term follow-up indicate that patients continue to die at a high rates after surviving hospitalisation for acute respiratory distress syndrome (ARDS, a severe form of AHRF), ${ }^{34}$ but without a comparison cohort, we cannot determine whether this late mortality is the result of pre-existing

\section{Key messages}

What is the key question?

- To examine whether late mortality after acute hypoxic respiratory (AHRF) is predominantly driven by pre-existing comorbid disease, the acute inciting event that precipitates respiratory failure (pneumonia, non-pulmonary infection, aspiration, trauma, pancreatitis) or the development of respiratory failure itself.

\section{What is the bottom line?}

- One in four survivors of AHRF has a late death that is not explained by pre-AHRF health status, but $70 \%$ of this increased risk for late death is due to the acute inciting events, while only $30 \%$ is due to the development of respiratory failure itself.

Why read on?

- We studied a large national cohort with longitudinal data to examine late mortality after AHRF hospitalisation relative to two carefully matched control groups-patients hospitalised with acute events that may precipitate AHRF and non-hospitalised adults.

comorbid disease versus a direct effect of AHRF. An older study suggests that patients surviving ARDS do not have increased risk of late mortality relative to patients surviving hospitalisation for inciting events alone-but was not powered to detect effects smaller than a $14 \%$ absolute difference. ${ }^{5}$ With declining in-hospital mortality, ${ }^{6-8}$ AHRF-related mortality may now be shifted to the posthospital period. Other critical illnesses, such as sepsis ${ }^{9}$ and delirium, ${ }^{10}$ are associated with increased late mortality. Thus, we hypothesised that AHRF may also increase patients' risk for late mortality.

Understanding whether patients who survive AHRF acutely face increased risk for late death is important for (1) measuring the overall burden of AHRF-related mortality, (2) determining whether AHRF is associated with prolonged pathobiological derangements that may be amenable to treatment, (3) informing the timing of interventions to improve long-term survivorship after AHRF, (4) selecting clinical trial end-points and (5) understanding the prognosis of patient surviving AHRF.

To address these questions, we compared patients hospitalised with AHRF to two controls: adults not currently hospitalised and patients hospitalised with acute inciting events that may result in AHRF-pneumonia, non-pulmonary infection, 
aspiration, trauma or pancreatitis. These comparisons allow us to determine both (1) the excess late mortality of an episode of AHRF and (2) the incremental excess late mortality of AHRF, above and beyond what is associated with hospitalisation for acute inciting events.

\section{METHODS}

\section{Study population}

We studied participants in the US Health and Retirement Study (HRS), a longitudinal cohort of 37000 adults aged 51 and above in 23000 households, ongoing since $1992 .{ }^{11}$ The study uses a multistage probability sample to identify participants. ${ }^{11}$ The sociodemographic and racial distribution is broadly representative of the older US population. ${ }^{12-14}$ The cohort is interviewed every 2 years with a follow-up rate consistently over $90 \% .^{11}$ Survey questions focus on wealth, health, cognition and employment. ${ }^{11}$ Data are linked to federal health insurance (Medicare) claims. ${ }^{11}$ Participants provide informed consent on enrolment to HRS and again for linkage to Medicare insurance claims. We considered fee-for-service Medicare beneficiaries aged $\geq 65$ who took part in at least one survey during 1998-2010 for inclusion in the study.

\section{Study cohorts}

We identified three study cohorts. The primary cohort was patients hospitalised with AHRF. The comparison cohorts were (1) adults not currently in the hospital (non-hospitalised adults) and (2) patients hospitalised with an acute inciting event that may cause AHRF (at-risk hospitalisations).

We defined AHRF hospitalisations pragmatically in administrative claims as (1) diagnostic codes for one or more acute inciting events that may result in AHRF-pneumonia, non-pulmonary infection, aspiration, trauma or pancreatitis and (2) respiratory failure-a procedural code for invasive mechanical ventilation and/or an explicit diagnosis code of acute respiratory failure (online supplementary table S1). We validated this claimsbased AHRF definition in the University of Michigan Health System. It had a positive predictive value of $81.4 \%$ (95\% CI $72.3 \%$ to $88.6 \%)$ and a negative predictive value of $91.9 \%(95 \%$ CI $90.0 \%$ to $93.4 \%$ ) when compared with a clinical AHRF definition of $\mathrm{PaO}_{2} / \mathrm{FIO}_{2}<300$ while on $\geq 40 \%$ fraction of inspired oxygen (online supplementary appendix 1 ).

We defined at-risk hospitalisations as those with diagnostic codes for one or more acute inciting events that may result in AHRF, but no diagnostic code for either respiratory failure or mechanical ventilation. We excluded hospitalisations with diagnostic codes for stroke or chronic respiratory failure from both hospitalised cohorts, as these diagnoses suggest a cause for respiratory failure other than an acute pulmonary process.

The cohort of non-hospitalised adults was not in the hospital at the time point that they were matched, but no exclusions were placed on this cohort being admitted to the hospital either before or after the date of match. (Further details of this matching approach are described in a previous publication. ${ }^{9}$ )

\section{Matching}

At the time of each HRS survey, we estimated each participant's risk of having an AHRF hospitalisation in the next 2 years using multiple logistic regression. We selected the following predictor variables based on previous research and/or clinical experience suggesting it is an important risk factor for AHRF, or acute hospitalisation $^{15}$
- Demographics: age, self-reported race and ethnicity, gender, partnership status.

- Economic status: wealth (sum of all assets and debts) standardised to 2013 \$ using the annual gross domestic product price index, ${ }^{16}$ previous or current use of food stamps (a government assistance programme for low-income families and individuals).

- Healthcare utilisation: number of hospitalisations in the prior year, AHRF hospitalisation in the prior year, residence in a nursing home.

- Health status: limitations of five activities and six instrumental activities of daily living (IADLs/ADLs), self-rating of health, body mass index (BMI).

\section{- Comorbidity burden: 17 Charlson comorbidities. ${ }^{17} 18$}

Limitations of ADLs, IADLs, self-reported health, government assistance and $\mathrm{BMI}$ were missing in $5.6 \%, 0.1 \%, 0.1 \%, 0.5 \%$ and $1.4 \%$, respectively, and these values were imputed using multiple imputation with chained equations and five imputations. ${ }^{19}$ Other covariates were present for the entire population. We considered select interactions (gender and partnership) and non-linear forms (age) based on a priori knowledge of the relations between the predictors and the likelihood of hospitalisation. ${ }^{20}$

We matched patients in the AHRF cohort 1:1 to patients in the at-risk hospitalisation cohort by age, percentile risk for AHRF hospitalisation, number of hospitalisations in the year prior to index admission, AHRF hospitalisation in the prior year, acute precipitating events during index hospitalisation (pneumonia, non-pulmonary infection, aspiration, trauma and pancreatitis) and other conditions during the index hospitalisation that may contribute to respiratory failure (congestive heart failure and asthma/chronic obstructive pulmonary disease) using coarsened exact matching. ${ }^{21}$

We examined the balance of each covariate (those directly matched, as well as those included within the multivariable risk for AHRF hospitalisation) between the hospitalised cohorts using $\mathrm{X}^{2}$ and $\mathrm{t}$ tests, as appropriate, without consideration of any outcome. ${ }^{22}$ We then re-matched the hospitalised cohorts including any variables that were unbalanced on the initial match. We then re-examined and confirmed covariate balance before examining any results. We matched patients in the AHRF cohort 1:1 in adults in the non-hospitalised cohort again using an iterative process to ensure covariate balance. Further details are provided in the online supplementary appendix 2 .

\section{Outcomes and statistical analysis}

Our primary outcome of interest was late mortality, defined as death in the 31 days to 2 years following AHRF hospital admission, at-risk hospital admission or the date of match, depending on the cohort. All patients were followed to death, or for 2 years. We used multivariable Cox proportional hazards models to calculate the HR for late mortality, adjusted for age, gender and propensity of AHRF. In addition, we used multivariable logistic regression models in order to calculate adjusted proportions of mortality. The technique of matching patients, then also adjusting for potential confounders using regression, is known as 'doubly-robust' estimation because it combines two methods for reducing bias and is less sensitive to misspecification. ${ }^{23}{ }^{24}$ In the primary analysis, we accounted for the clustering of hospitalisations within people using clustered robust standard errors. ${ }^{25}$ This approach corrects standard errors so that significance tests, which assume independence of observations, are still valid.

Beyond measuring risk of late mortality overall, we also measured risk of death at multiple time intervals (0-30 days, 
31-90 days, 181 days-1 year and 1-2 years) to examine how long excess mortality persists after AHRF. In addition, we present the risk of mortality over time in a hazard plot. To estimate the proportion of late mortality due to hospitalisation for the acute inciting invent(s) versus the development of AHRF, we examined the ratio of logistic regression coefficients among AHRF hospitalisations that were matched to both controls (as described in the online supplementary appendix 2).

We performed several exploratory analyses. First, we examined whether excess late mortality after AHRF differed by type of acute inciting event, age, sex, self-rating of health, comorbidity burden, pre-AHRF functional limitations and nursing home residence. Second, we examined risk of late death separately for earlier (19982005) versus later hospitalisations (2006-2012), since management of AHRF has changed over time. Third, we examined risk of late death among patients with no hospitalisation for AHRF in the prior year. Fourth, we examined the proportion of patients surviving to 31 days who had a subsequent hospitalisation, as well as the subset who survived to 2 years. Fifth, we examined the principal diagnosis category $^{26}$ of the final hospitalisations (a proxy for cause of death ${ }^{27}$ ) among patients with AHRF with a late death.

We performed five sensitivity analyses, described in the online supplementary appendix 2. Conceptually, sensitivity analyses 1-3 used alternative approaches to account for clustering of observations within people, since each person may have multiple hospitalisations and/or times of non-hospitalisation. Sensitivity analyses 4 and 5 used alternate approaches to matching. We conducted all analyses with Stata MP V. 14 (StataCorp, College Station, TX). We used two-sided hypothesis testing and set significance at $\mathrm{p}<0.05$.

\section{RESULTS}

From 15075 HRS participants with Medicare linkage and at least one survey completed in 1998-2010, we identified 1268
AHRF hospitalisations, 13117 at-risk hospitalisations and 15033 non-hospitalised adults (figure 1 ).

For the 1238 AHRF hospitalisations, patients were elderly (median age 79), 54.5\% were female, $80.2 \%$ were white, $11.2 \%$ were nursing home residents, with a median of one functional limitations and two medical comorbidities (table 1). Over $60 \%$ of patients rated their health as fair or poor. Risk factors for AHRF were non-pulmonary infection (65.4\%), pneumonia (45.0\%), aspiration (18.0\%), trauma (5.6\%) and pancreatitis (1.3\%). 33.5\% had multiple risk factors.

Mortality was high: $42.7 \%$ at 30 days, $65.5 \%$ at 1 year and $73.3 \%$ at 2 years. Among the 727 patients who survived to day 31, 131 (18.0\%) died by 90 days and 389 (53.5\%) died by 2 years. Of the 389 patients who died during day 31 to 2 years, all but $23(5.9 \%)$ were discharged from the hospital prior to death.

We were able to match 1157 AHRF hospitalisations (91.5\% of all AHRF hospitalisations) to non-hospitalised adults, and 1017 AHRF hospitalisations (80.2\%) to at-risk hospitalisations (figure 1, online supplementary tables S2 and S3). Nine hundred and fifty-two AHRF hospitalisations (75.1\%) were matched to both an at-risk hospitalisation and a non-hospitalised adult; mortality for these double-matched cohorts is presented in online supplementary table S4. AHRF was associated with markedly increased acute mortality relative to matched non-hospitalised adults (adjusted HR (aHR) for 30-day mortality: 30.5; 95\% CI 19.8 to 47.1) and matched at-risk hospitalisations (aHR for 30-day mortality: 4.0; 95\% CI 3.3 to 4.8 ). The risk of mortality waned with time (figure 2), becoming equivalent to at-risk hospitalisation around day 90 . However, risk remained slightly elevated compared with non-hospitalised controls to beyond 1 year.

Compared with adults not in the hospital matched on all baseline characteristics, patients with AHRF experienced a $24.4 \%$ (95\% CI $19.9 \%$ to $28.9 \%$ ) absolute increase (or 1.9-fold

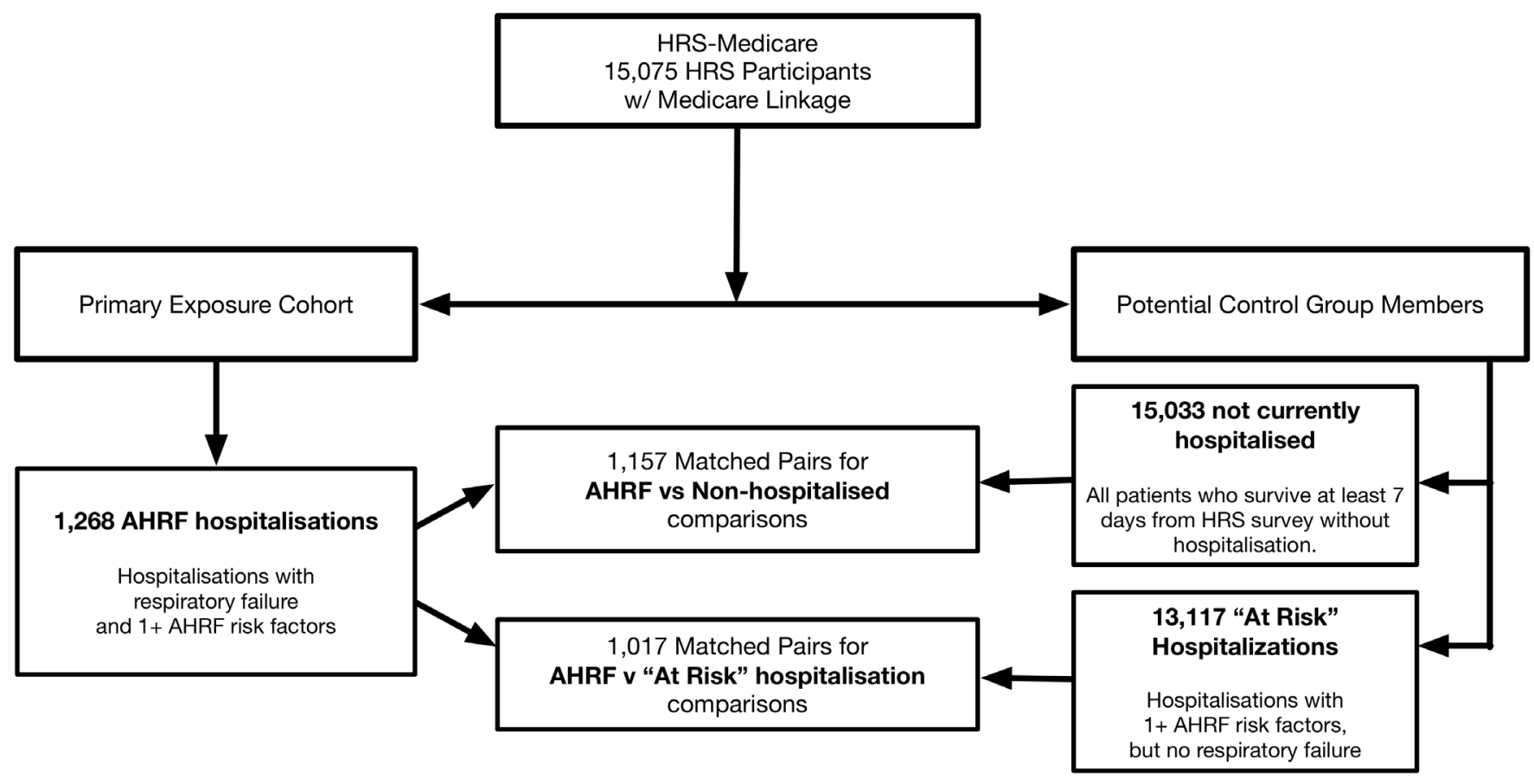

Figure 1 Cohort flow. 
Table 1 Baseline characteristics of AHRF cohort $(n=1268)$

\begin{tabular}{|c|c|}
\hline \multicolumn{2}{|l|}{ Demographics } \\
\hline Age, years, median (IQR) & $79(72-85)$ \\
\hline Male, n (\%) & $589(46.5 \%)$ \\
\hline \multicolumn{2}{|l|}{ Race, n (\%) } \\
\hline White/Caucasian & $1017(80.2 \%)$ \\
\hline Black/African American & $220(17.4 \%)$ \\
\hline Other & $31(2.4 \%)$ \\
\hline Hispanic, n (\%) & $96(8.4 \%)$ \\
\hline Married or partnered, $\mathrm{n}(\%)$ & $591(46.6 \%)$ \\
\hline \multicolumn{2}{|l|}{ Economic status } \\
\hline \multicolumn{2}{|l|}{ Total wealth } \\
\hline Quintile 5 positive assets & $172(13.7 \%)$ \\
\hline Quintile 4 positive assets & $186(14.7 \%)$ \\
\hline Quintile 3 positive assets & $186(14.7 \%)$ \\
\hline Quintile 2 positive assets & $242(19.2 \%)$ \\
\hline Quintile 1 positive assets & $324(25.7 \%)$ \\
\hline Net negative or zero assets & $150(11.9 \%)$ \\
\hline Government assistance & $120(9.6 \%)$ \\
\hline \multicolumn{2}{|l|}{ Pre-AHRF health status } \\
\hline Charlson Comorbidity Index, median (IQR) & $2(0-4)$ \\
\hline Congestive heart failure & $387(30.5 \%)$ \\
\hline Dementia & $97(7.7 \%)$ \\
\hline IADL/ADL limitations, median (IQR) & $1(0-5)$ \\
\hline \multicolumn{2}{|l|}{ Self-rating of health, $\mathrm{n}(\%)$} \\
\hline Excellent & $31(2.5 \%)$ \\
\hline Very good & $150(11.9 \%)$ \\
\hline Good & $300(23.7 \%)$ \\
\hline Fair & $391(30.9 \%)$ \\
\hline Poor & $394(31.1 \%)$ \\
\hline \multicolumn{2}{|l|}{ Body mass index, $\mathrm{n}(\%)$} \\
\hline Very severely obese & $57(4.6 \%)$ \\
\hline Severely obese & $73(5.9 \%)$ \\
\hline Obese & $164(13.2 \%)$ \\
\hline Overweight & $355(28.6 \%)$ \\
\hline Normal & $499(40.2 \%)$ \\
\hline Underweight & $94(7.6 \%)$ \\
\hline Multivariable risk for AHRF & $5.9 \%(3.4 \%-10.7 \%)$ \\
\hline \multicolumn{2}{|l|}{ Pre-AHRF healthcare use } \\
\hline Hospitalisations in prior year, median (IQR) & $1(0-2)$ \\
\hline AHRF in prior year, $\mathrm{n}(\%)$ & $151(11.9 \%)$ \\
\hline Residence in a nursing home, $\mathrm{n}(\%)$ & $142(11.2 \%)$ \\
\hline \multicolumn{2}{|l|}{ Hospitalisation diagnoses } \\
\hline Risk factor for direct AHRF & $766(60.4 \%)$ \\
\hline Pneumonia & $570(45.0 \%)$ \\
\hline Aspiration & $228(18.0 \%)$ \\
\hline Risk factor for indirect AHRF & $890(70.2 \%)$ \\
\hline Non-pulmonary infection & $834(65.8 \%)$ \\
\hline Trauma & $71(5.6 \%)$ \\
\hline Pancreatitis & $16(1.3 \%)$ \\
\hline
\end{tabular}

AHRF, acute hypoxic respiratory failure; IADL, instrumental activities of daily living. relative increase) in late mortality. Adjusted 31-day to 2-year mortality was $52.4 \%$ (95\% CI $48.5 \%$ to $56.2 \%)$ in patients with AHRF surviving to at least 31 days versus 28.0\% (95\% CI $25.4 \%$ to $30.6 \%$ ) among matched non-hospitalised patients who survived to at least 31 days (aHR for late mortality: 2.5 , $\mathrm{p}<0.001$ ) (figure $3 \mathrm{a}$, online supplementary table 5). Adjusted 91-day to 2 -year mortality was $42.0 \%$ (95\% CI $37.9 \%$ to 46.2\%) in survivors with AHRF versus 25.9\% (95\% CI 23.3\% to $28.6 \%$ ) in matched non-hospitalised patients, $\mathrm{p}<0.001$. The higher mortality in the AHRF cohort persisted for the full 2-year follow-up period. Among patients with AHRF who survived to 1 year, the adjusted 2-year mortality was $21.0 \%$ (95\% CI $17.0 \%$ to $25.0 \%$ ) versus $14.9 \%$ (95\% CI $12.6 \%$ to $17.2 \%$ ) in their controls who were not in the hospital at the time of matching (aHR for 2-year mortality: 1.4, $\mathrm{p}=0.006$ ).

Compared with matched patients hospitalised with the same acute inciting event, patients with AHRF experienced a 6.7\% (95\% CI $1.7 \%$ to $11.7 \%)$ absolute increase (1.15-fold relative increase) in late mortality (aHR for late mortality: 1.2, $\mathrm{p}=0.01$ ) (figure $3 \mathrm{~b}$, online supplementary table S5). The increased risk for late mortality did not persist beyond 90 days. Among those who survived to at least 91 days, risk mortality at 2 years was indistinguishable from patients surviving hospitalisation for AHRF risk factors alone ( $\mathrm{aHR}$ for 2-year mortality $=1.1, \mathrm{p}=0.23$ ).

Among patients with AHRF who survived to day 31 and were matched to both an at-risk hospitalisation and a non-hospitalised adult, at-risk hospitalisation accounted for $71.2 \%$ (95\% CI $52.7 \%$ to $89.6 \%$ ) of the increased odds of late mortality, whereas the development of AHRF itself accounted for $28.8 \%(95 \% \mathrm{CI}$ $10.4 \%$ to $47.3 \%$ ) of the increased odds of late mortality.

In all five sensitivity analyses, the point estimates of ORs for late mortality relative to non-hospitalised adults and relative to patients with an at-risk hospitalisation were similar to the ORs in the primary analyses (online Supplementary table S6). Statistical significance was lost, however, in two instances due to the smaller sample sizes in the sensitivity analysis. Results were also similar for earlier versus later hospitalisations.

In stratified analysis, the excess late (31 days to 2 years) mortality after AHRF was relatively constant across patient subgroups defined by type of acute inciting event, sex, comorbidity burden, functional limitations, self-rating of health and nursing home residence (Figure 4 online supplementary figure S1). However, the incremental effect of AHRF versus at-risk hospitalisation on late mortality was greater in patients $\geq 80$ years versus patients $<80$ years (figure 4 ). Risk for late death was similar when limiting the analysis to earlier hospitalisation (1998-2005), later hospitalisation (2006-2012)and patients without a hospitalisation for AHRF in the prior year (online supplementary table S7 and S8).

Re-hospitalisation was more common in AHRF versus comparison cohorts (online supplementary table S9 and figure S2). Of the 398 patients with AHRF who experienced a late death following AHRF, 290 (72.9\%) were re-hospitalised prior to their death. The most common hospitalisation diagnoses prior to death were sepsis $(16.6 \%$ of terminal hospitalisations), respiratory failure (15.2\%), congestive heart failure $(9.0 \%)$, aspiration pneumonitis $(6.6 \%)$ and pneumonia (5.2\%).

\section{DISCUSSION}

In this national cohort of older Americans, late mortality was substantially increased after AHRF relative to non-hospitalised adults. Among patients who survived to at least day 31 after hospital 


\section{Risk of Death over Time}
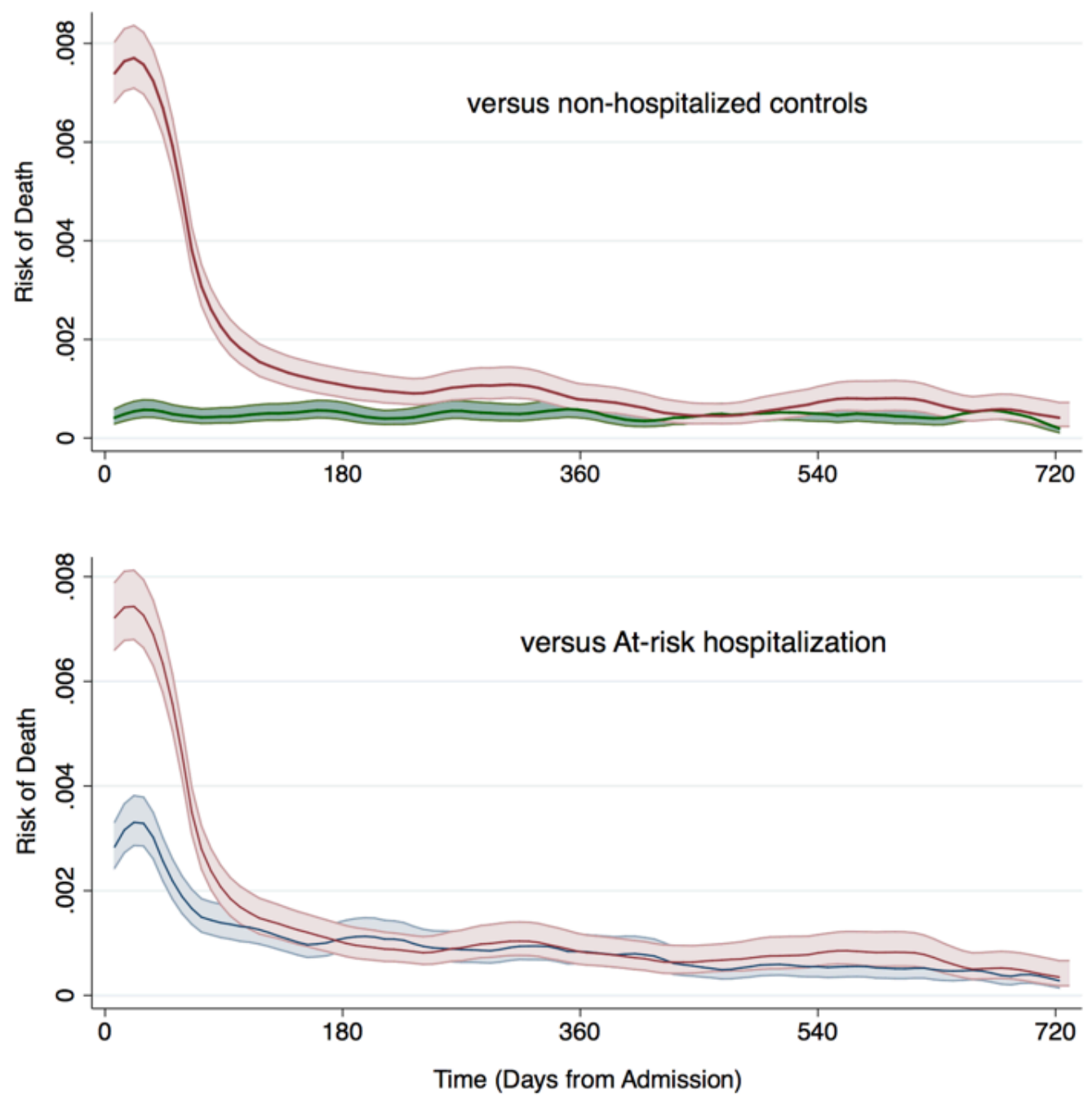

Figure 2 Risk of death over time. The risk of death over time is shown for the AHRF cohort (in red), non-hospitalised cohort (in green) and at-risk cohort (in blue). The shaded area represents the $95 \% \mathrm{CI}$. AHRF, acute hypoxic respiratory failure.

admission for AHRF, more than half died within the next 2 years. This high rate of late mortality was not explained by age, sociodemographics or health status prior to AHRF. Compared with adults not in the hospital who were indistinguishable on a variety of potential confounders prior to acute illness, patients surviving AHRF have a nearly $25 \%$ absolute increase in late mortality.

The majority $(70 \%)$ of the late mortality after AHRF was explained by hospitalisation for the acute inciting event(s), while a minority (30\%) was explained by the development of AHRF. Compared with patients hospitalised with acute inciting event(s) but without AHRF, those with AHRF had a 7\% absolute increase in late mortality. Among hospitalised patients who survived for at least 90 days; however, the risk for subsequent mortality was indistinguishable. Subgroup analysis suggested that the incremental effect of AHRF on late mortality may be concentrated in the oldest patients.

Prior studies have shown that ARDS is associated with significantly increased early (30 day) mortality compared with hospitalisation for inciting events that may lead to ARDS, such as hospitalisation for sepsis ${ }^{28}$ and trauma. ${ }^{29}$ Our findings on early mortality are similar. Compared with patients who were hospitalised for acute inciting events alone, AHRF was associated with a fivefold relative increase, or approximately 30\% absolute increase in 30-day mortality in our study. This can be considered as a form of 'positive control', in that it confirms that our AHRF population is distinct from the non-AHRF hospitalised patients.

We were able to identify just one prior study that examined late mortality of survivors with ARDS or AHRF relative to matched controls. This study of patients treated in the mid-1990s found no difference in late mortality between 127 survivors with ARDS and 127 controls matched by acute inciting event (sepsis or trauma), illness severity and admission half-year. ${ }^{5}$ However, the study had limited power, and so an important difference in late mortality (as large as 13\%) could not be ruled out. With a larger sample, our study was able to detect a small increase in risk of late mortality after AHRF relative to hospitalisations for acute inciting events alone, but approximately 70\% the excess risk for late mortality after AHRF was explained by at-risk hospitalisation.

While it may seem counterintuitive for survivors with AHRF to experience similar late mortality as survivors of less severe illness, this phenomenon has precedent. Elderly survivors of in-hospital cardiac arrest have only modestly lower 2-year 

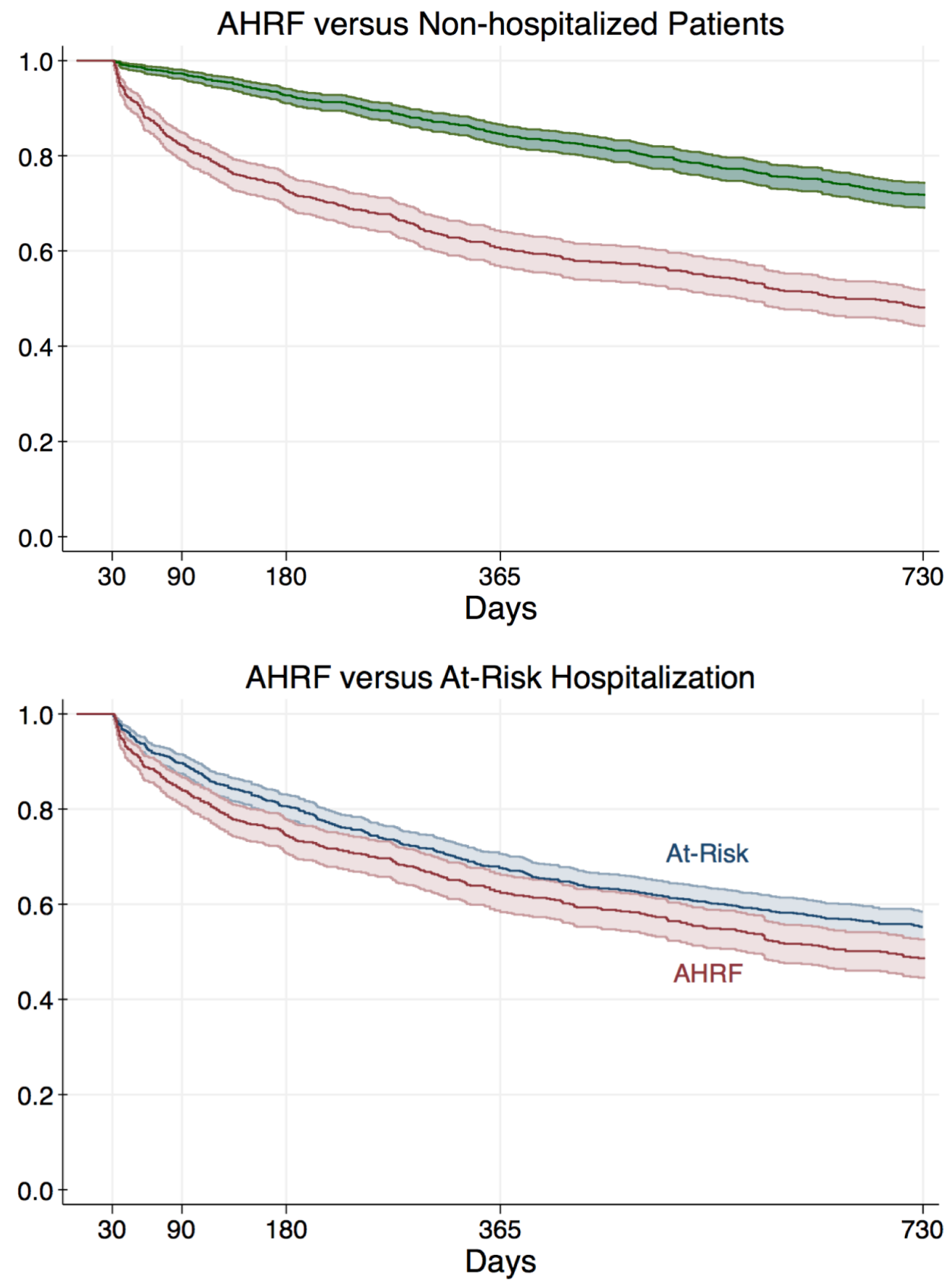

Figure 3 Kaplan-Meier survival curves for AHRF cohorts versus matched comparison cohorts showing long-term survival of patients who survived at least 30 days after this match day. The proportion surviving over time is shown for the AHRF cohort (in red), non-hospitalised cohort (in green) and at-risk cohort (in blue). The shaded area represents the 95\% Cl.AHRF, acute hypoxic respiratory failure.

survival rates compared with patients who survive a hospitalisation for heart failure, and the survival curves converge at 3 years ${ }^{30}$.

The contrast between the present results showing a small degree of excess late mortality after AHRF relative to matched hospitalised controls and previous work using a similar methodology for late mortality after sepsis ${ }^{9}$ is intriguing. It raises the possibility that our focus on mechanical ventilation as the key risk factor in mediating late harm in patients surviving the ICU may be too narrow. For example, mechanical ventilation is often a key inclusion criteria for post-ICU rehabilitation studies, ${ }^{31}$ but may not target the most appropriate population. The contrast also suggests that the inflammation and multi-organ failure associated with ventilator-induced lung injury ${ }^{32}$ may resolve faster than the inflammation and immune-suppression that follows sepsis. ${ }^{33}$ Future epidemiological and translational studies are needed to better understand the duration and recovery of these pathobiological derangements-and how the natural history differs between AHRF and sepsis.

Our study should be interpreted in the context of several limitations. First, this is an observational study, so cannot prove causation. We have attempted to control for confounding as best as possible through matching and regression adjustment, as well as by studying a cohort with prospectively collected data on a range of potential confounders. Second, we ascertained AHRF by International Classification of DIseases, Ninth Revision, 


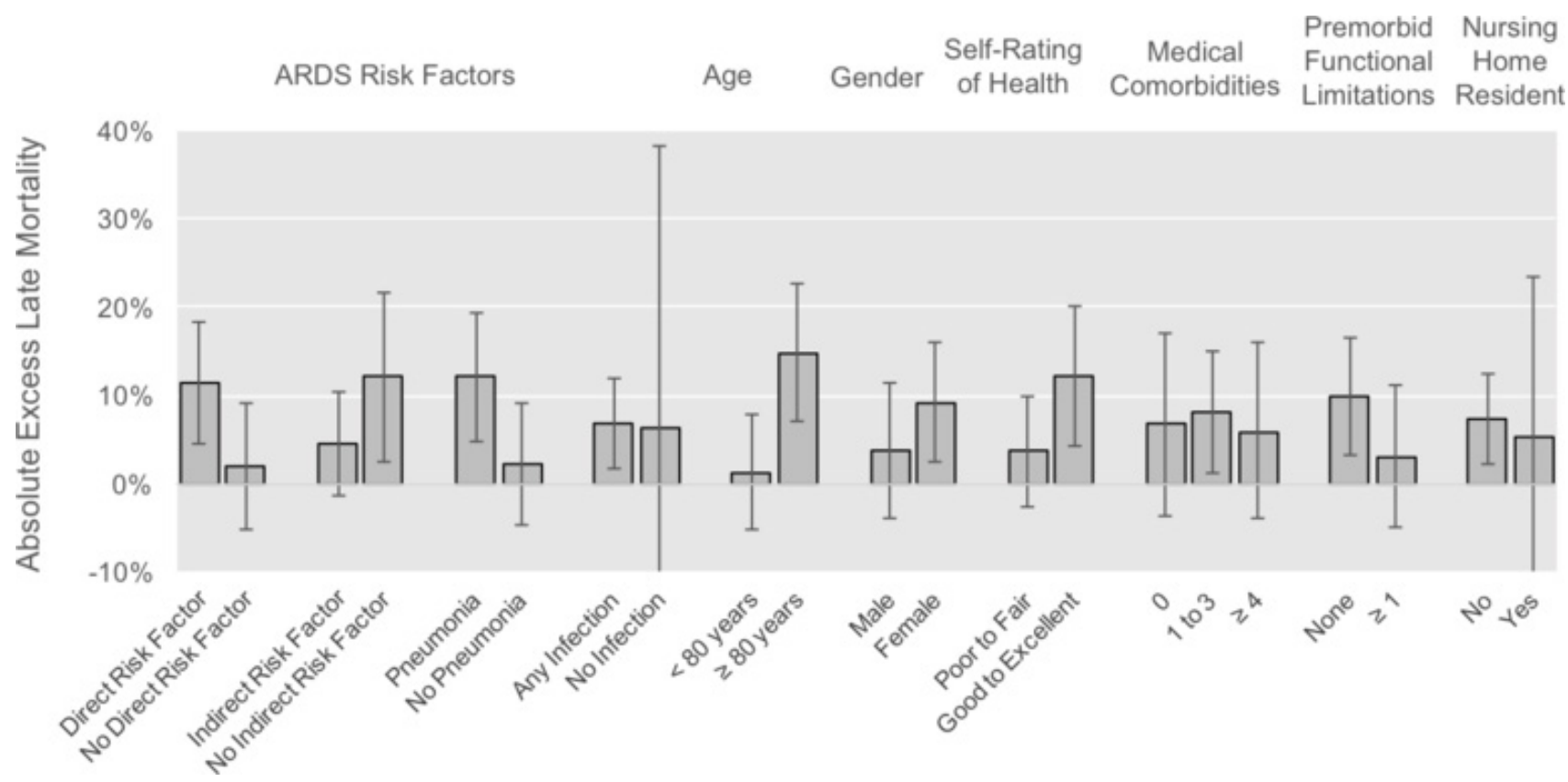

Figure 4 Absolute excess late mortality of AHRF versus at-risk hospitalisations, stratified by subgroup. AHRF, acute hypoxic respiratory failure.

Clinical Modification (ICD-9-CM) coding for acute inciting events and respiratory failure. There may have been misclassification in both directions between at-risk and AHRF hospitalisations, but the negative and positive predictive values from our validation suggest that the degree of misclassification is likely small. Third, our study spanned a period of 15 years when treatment for AHRF changed. However, when limiting our analysis to only early or late hospitalisations, the associations were similar. Fourth, we were not able to examine the subset of patients with AHRF and ARDS because there is no reliable way to identify ARDS in administrative claims. Nonetheless, we believe AHRF to be a clinically meaningful study population, ${ }^{34}$ and postulate that our findings are reflective of ARDS patients as well. Fifth, we excluded patients aged $<65$ years because our study relied on Medicare claims, and only limited groups aged under 65 qualify for Medicare coverage.

Our study has several strengths. First, we believe that it is the only recent study to examine the excess late mortality associated with AHRF. Second, by examining a national cohort with detailed survey and claims data, we were able to achieve robust control for confounding. Third, by comparing patients with AHRF with two controls, we were able to disentangle the proportion of excess mortality related to hospitalisations for acute inciting events versus the development of AHRF. Finally, our findings were robust to several sensitivity analyses.

\section{CONCLUSION}

In a national cohort of older Americans, we have shown that AHRF is associated with a high rate of late mortality that is not explained by health status before AHRF. However, more than $70 \%$ of the excess risk for late mortality is explained by hospitalisation for an acute inciting event, not hypoxemic respiratory failure.

Acknowledgements The authors appreciate the expert programming of Ryan McCammon, MS, and Vanessa Dickerman, MS, at the University of Michigan, Ann Arbor. They were not compensated for their contributions besides salary. The authors thank the participants in the Health and Retirement Study whose data were included in this study.
Contributors HP designed the study, analysed the data, interpreted the data and drafted the manuscript. DFM and TJI designed the study, interpreted the data and edited the manuscript for critical intellectual content. MWS and KML interpreted the data and edited the manuscript for critical intellectual content. TJI and DFM contributed equally.

Funding This work was supported by grants K08 GM115859 [HCP] and T32HL007749 [MWS] from the National Institutes of Health. The Health and Retirement Study is sponsored by the National Institute on Aging (U01 AG009740) and performed at the Institute for Social Research, University of Michigan.

Disclaimer The views expressed in this article are those of the authors and do not necessarily reflect the position or policy of the Department of Veterans Affairs or the US government.

Competing interests None declared.

Ethics approval University of Michigan IRB approved this study. Informed consent was obtained on enrolment into Health and Retirement Study and again for Medicare linkage.

Provenance and peer review Not commissioned; externally peer reviewed.

Open access This is an open access article distributed in accordance with the Creative Commons Attribution Non Commercial (CC BY-NC 4.0) license, which permits others to distribute, remix, adapt, build upon this work non-commercially, and license their derivative works on different terms, provided the original work is properly cited and the use is non-commercial. See: http://creativecommons.org/ licenses/by-nc/4.0/

(c) Article author(s) (or their employer(s) unless otherwise stated in the text of the article) 2018. All rights reserved. No commercial use is permitted unless otherwise expressly granted.

\section{REFERENCES}

1 Vincent JL, Akça S, De Mendonça A, et al. The epidemiology of acute respiratory failure in critically ill patients $\left({ }^{*}\right)$. Chest 2002;121:1602-9.

2 Bellani G, Laffey JG, Pham T, et al. Epidemiology, patterns of Care, and mortality for patients with acute respiratory distress syndrome in Intensive Care Units in 50 countries. JAMA 2016;315:788-800.

3 Needham DM, Colantuoni E, Mendez-Tellez PA, et al. Lung protective mechanical ventilation and two year survival in patients with acute lung injury: prospective cohort study. BMJ 2012;344:e2124.

4 Wang $C$, Calfee CS, Paul DW, et al. One-year mortality and predictors of death among hospital survivors of acute respiratory distress syndrome. Intensive Care Med 2014:40:388-96.

5 Davidson TA, Rubenfeld GD, Caldwell ES, et al. The effect of acute respiratory distress syndrome on long-term survival. Am J Respir Crit Care Med 1999;160:1838-42. 
6 Zambon M, Vincent JL. Mortality rates for patients with acute lung injury/ARDS have decreased over time. Chest 2008;133:1120-7.

7 Erickson SE, Martin GS, Davis JL, et al. Recent trends in acute lung injury mortality: 1996-2005. Crit Care Med 2009:37:1574-9.

8 Sigurdsson MI, Sigvaldason K, Gunnarsson TS, et al. Acute respiratory distress syndrome: nationwide changes in incidence, treatment and mortality over 23 years. Acta Anaesthesiol Scand 2013;57:37-45.

9 Prescott HC, Osterholzer JJ, Langa KM, et al. Late mortality after sepsis: propensity matched cohort study. BMJ 2016;353:i2375.

10 Ely EW, Shintani A, Truman B, et al. Delirium as a predictor of mortality in mechanically ventilated patients in the intensive care unit. JAMA 2004:291:1753.

11 Sonnega A, Faul JD, Ofstedal MB, et al. Cohort Profile: the Health and Retirement Study (HRS). Int J Epidemiol 2014;43:576-85.

12 Meijer E, Karoly LA. Representativeness of the Low-Income Population in the Health and Retirement Study, 2013.

13 Ofstedal MB, Weir DR. Recruitment and retention of minority participants in the health and retirement study. Gerontologist 2011:51(Suppl 1):S8-20.

14 Levy H, Gutierrez I. Documentation and benchmarking of health insurance measures in the Health and Retirement Study, 2009.

15 Kansagara D, Englander H, Salanitro A, et al. Risk prediction models for hospital readmission: a systematic review. JAMA 2011;306:1688-98.

16 U.S. Department of Commerce. Bureau of Economic Analysis Website. 2014 http:// bea.gov/iTable/iTable.cfm?Req $\mid \mathrm{D}=9 \&$ step $=1$ \#reqid $=9 \&$ step $=1$ \&isuri $=1$

17 Deyo RA, Cherkin DC, Ciol MA. Adapting a clinical comorbidity index for use with ICD-9-CM administrative databases. J Clin Epidemiol 1992;45:613-9.

18 Klabunde CN, Potosky AL, Legler JM, et al. Development of a comorbidity index using physician claims data. J Clin Epidemiol 2000;53:1258-67.

19 Royston P. Multiple imputation of missing values. Stata J 2004:4:227-41.

20 Steyerberg, E. W. \& SpringerLink (Online service). Clinical Prediction Models A402 Practical Approach to Development, Validation, and Updating, 2009.

21 lacus SM, King G, Porro G. Cem : Software for Coarsened Exact Matching. I Stat Softw 2009:30 http:///.mp/Te8KP5
22 Rubin DB. For objective causal inference, design trumps analysis. Ann App/ Stat 2008:2:808-40.

23 Funk MJ, Westreich D, Wiesen C, et al. Doubly robust estimation of causal effects. Am J Epidemiol 2011;173:761-7.

24 Rubin DB. Using multivariate matched sampling and regression Adjustment to control Bias in Observational Studies. J Am Stat Assoc 1979:74:318-28.

25 Williams RL. A note on robust variance estimation for cluster-correlated data. Biometrics 2000:56:645-6.

26 HCUP CCS. Healthcare Cost and Utilization Project (HCUP). Agency for Healthcare Research and Quality, Rockville, MD. 2014 www.hcup-us.ahrq.gov/toolssoftware/ccs/ ccs.jsp

27 Govindan S, Shapiro L, Langa KM, et al. Death certificates underestimate infections as proximal causes of death in the U.S. PLoS One 2014:9:e97714.

28 Mikkelsen ME, Shah CV, Meyer NJ, et al. The epidemiology of acute respiratory distress syndrome in patients presenting to the emergency department with severe Sepsis. Shock 2013;40:375-81.

29 Shah $\mathrm{CV}$, Localio AR, Lanken PN, et al. The impact of development of acute lung injury on hospital mortality in critically ill trauma patients. Crit Care Med 2008;36:2309-15.

30 Chan PS, Nallamothu BK, Krumholz HM, et al. Long-term outcomes in elderly survivors of in-hospital cardiac arrest. N Eng/ J Med 2013;368:1019-26.

31 Mehlhorn J, Freytag A, Schmidt K, et al. Rehabilitation interventions for postintensive care syndrome: a systematic review. Crit Care Med 2014;42:1263-71.

32 Plötz FB, Slutsky AS, van Vught AJ, et al. Ventilator-induced lung injury and multiple system organ failure: a critical review of facts and hypotheses. Intensive Care Med 2004;30:1865-72.

33 Hotchkiss RS, Monneret G, Payen D. Sepsis-induced immunosuppression: from cellular dysfunctions to immunotherapy. Nat Rev Immunol 2013;13:862-74.

34 Luhr OR, Antonsen K, Karlsson M, et al. Incidence and mortality after acute respiratory failure and acute respiratory distress syndrome in Sweden, Denmark, and Iceland. The ARF Study Group. Am J Respir Crit Care Med 1999;159:1849-61. 